\title{
A search for transiting extrasolar planet candidates in the OGLE-II microlens database of the galactic plane
}

\author{
I. A. G. Snellen, R. F. J. van der Burg, M. D. J. de Hoon, and F. N. Vuijsje
}

Leiden Observatory, Leiden University, Postbus 9513, 2300 RA Leiden, The Netherlands

e-mail: snellen@strw.leidenuniv.nl

Received 27 July 2007 / Accepted 9 October 2007

\section{ABSTRACT}

\begin{abstract}
Context. In the late 1990s, the Optical Gravitational Lensing Experiment (OGLE) team conducted the second phase of their long-term monitoring programme, OGLE-II, which since has been superseded by OGLE-III. All the monitoring data of this second phase, which was primarily aimed at finding microlensing events, have recently been made public.

Aims. Fields in the OGLE-II survey have typically been observed with a cadence of once per night, over a period of a few months per year. We investigated whether these radically differently sampled data can also be used to search for transiting extrasolar planets, in particular in the light of future projects such as PanSTARRS and SkyMapper, which will monitor large fields, but mostly not at a cadence typical for transit studies.

Methods. We selected data for $\sim 15700$ stars with $13.0<I<16.0$ in three OGLE-II fields towards the galactic disc in the constellation Carina, each with 500-600 epochs of I-band photometry. These light curves were first detrended using Sys-Rem, after which they were searched for low amplitude transits using the Box Least Squares algorithm.

Results. The detrending algorithm significantly decreased the scatter in the light curves, from an average of $0.5 \%$ down to $0.2-0.3 \%$ for stars with $I<15$. Several dozens of eclipsing binaries and low amplitude transits were found, of which 13 candidates exhibit transits with such depth and duration that they are possibly caused by an object with a radius less than twice that of Jupiter. Eleven out of these thirteen candidates show significant ellipsoidal light variations and are unlikely to host a transiting extrasolar planet. However, OGLE2-TR-L9 (CAR_SC2_75679), is an excellent planet candidate comparable to the known OGLE-III transiting planets, and deserves further follow-up observations.
\end{abstract}

Key words. techniques: photometric - methods: data analysis - surveys - binaries: eclipsing - planetary systems

\section{Introduction}

An extrasolar planet that transits its host star is of great scientific value. Analysis of its transit light curve can reveal the planet/star size ratio, and implicitly the radius of the planet after the star has been spectrally typed. Since the occurrence of a transit implies that the inclination of the planetary orbit is near $90^{\circ}$, radial velocity measurements will reveal the true mass of the planet, in contrast to only a lower limit in mass for nontransiting planets. This will yield not only the mean density of the planet, but can also give insight into its internal structure (e.g. Sato et al. 2005). In addition, many interesting follow-up opportunities are within reach, such as atmospheric transmission spectroscopy, secondary eclipse and Rossiter-McLaughlin effect observations, and transit timing, providing information on the planet's atmosphere, orientation of its orbit, and on the presence of other planets in the system (e.g. Charbonneau et al. 2002; Charbonneau et al. 2005; Deming et al. 2005; Winn et al. 2007; Snellen 2004; Steffen et al. 2007).

Several successful transit surveys are now in place. OGLE-III (the third phase of the Optical Gravitational Lensing Experiment; Udalski et al. 2002a,b,c, 2004) is the longest running survey and has yielded so far 5 transiting planets. While more than 150 planet candidates have been reported by this team, most of them turned out to be blended or grazing eclipsing binaries and/or eclipsing M-dwarfs (Bouchy et al. 2005; Pont et al. 2005). It shows that radial velocity follow-up is crucial for the identification of the genuine transiting extrasolar planets. Now, several other dedicated ground-based transit surveys, such as XO (McCullough et al. 2005), SuperWASP (Collier Cameron et al. 2007), TrES (O'Donovan et al. 2007), and HAT (Bakos et al. 2007), have found their first planets. Therefore, the number of confirmed transiting planets has recently increased significantly (20 in total), with more than half of them discovered in the last 1.5 years. However, the number of planets found to transit the stars being monitored still lags behind the expectations from radial velocity surveys (Gaudi 2006). In addition, almost all of the planets found so far have radii larger than that of Jupiter. This indicates that a significant fraction of the transiting hot Jupiters are being missed. The most likely cause has been identified as correlated noise in the light curve data sets (e.g. Pont et al. 2006; Aigrain \& Pont 2007). Variations in atmospheric (seeing, airmass) or instrumental/telescope properties result in photometric features on the time scale of a transit, causing the sensitivity of a survey to decrease significantly. The recently launched CoRoT satellite and future Kepler mission will likely resolve these issues.

In this paper we present a search for transiting planet candidates in the 1997-2000 OGLE-II survey (Udalski et al. 1997), a precursor of OGLE-III, which was not designed for transit searches. While it is in itself highly desirable to find more transiting exoplanets, it is also valuable in the light of the radically different time sampling of OGLE-II compared to dedicated transit surveys (typically once per night compared to once per $15 \mathrm{~min}$.), which should significantly reduce correlated noise problems. This is particularly interesting in the light of future projects such 
Table 1. Details on the OGLE-II fields used in our analysis, with in Col. 1 the OGLE-II name, in Col. 2 the field center, Col. 3 the number of stars $13.0<I<16.0$, and in Col. 4 the number of epochs

\begin{tabular}{lcccc}
\hline \hline Name & $\begin{array}{c}\text { RA } \\
\text { h m }\end{array}$ & $\begin{array}{c}\text { Dec (J2000) } \\
\text { d m }\end{array}$ & $N_{\text {stars }}$ & $N_{\text {epochs }}$ \\
& 1106 & -6130 & 5531 & 502 \\
CAR_SC1 & 1106 & 562 & 562 \\
CAR_SC2 & 1108 & -6130 & 5265 & 485 \\
CAR_SC3 & 1110 & -6100 & 4915 & 480 \\
\hline
\end{tabular}

as PanSTARRS (e.g. Afonso \& Hennig 2007) and SkyMapper (e.g. Bayliss \& Sackett 2007); groundbased telescopes with huge field of view that will monitor millions of stars, but mostly at a cadence very different from transit surveys (although they will probably also spend some limited time performing transit surveys). In the next section the OGLE-II survey and our data analysis are presented. In Sect. 3 the results are shown and discussed, and our conclusions are given in Sect. 4. The folded light curves are presented in Fig. 4 in the appendix.

\section{OGLE-II survey and data analysis}

OGLE-II was the precursor of the current OGLE-III project, dedicated to microlensing work. All of its photometric data has been made available by the OGLE team on http://ogledb. astrouw.edu.pl/ ogle/photdb/ (Szymanski 2005). The data have been collected with the $1.3 \mathrm{~m}$ Warsaw Telescope at the Las Campanas observatory in Chile, using a $2048 \times 2048$ CCD camera in drift scan mode. Details on the instrumental setup can be found in Udalski et al. (1997). The observed fields cover $14.2 \times 57$ arcmin in the sky. All images were reduced by the OGLE team using the standard OGLE pipeline (Udalski et al. 1998). During the OGLE-II phase, fields have been observed towards the galactic bulge, the LMC and SMC, and the galactic disc. In this paper, we concentrate our analysis on the three galactic disc fields in the constellation Carina, which are the only disc fields which have been observed for more than 400 epochs, all taken between January 1997 and July 2000. The relevant field parameters are given in Table 1.

For our analysis we used the photometric data obtained from the Difference Image Analysis (DIA; Alard \& Lupton 1998; Alard 2000). We concentrated on those stars that have good data for more than $98 \%$ of the epochs, and downloaded all stars with a mean magnitude of $I<17.5$ (52703 stars in the three fields). In a later stage, our transit analysis only focuses on those stars with $13.0<I<16.0$ (15711 objects).

Sys-Rem, a detrending algorithm designed to remove systematic effects in a large set of light curves (Tamuz et al. 2005), was used on the data from the three fields. The algorithm can detect any systematic effect that appears linearly in many light curves obtained by the survey, without any prior knowledge of the origin of the effects. It has become a standard tool in transit survey light curve processing (e.g. Bakos et al. 2006; Collier Cameron et al. 2006). In Fig. 1 the scatter in the data is shown as a function of the mean stellar magnitude, for all stars in the CAR_SC1 field. The light-grey symbols indicate the dispersion before the use of Sys-Rem, with the black crosses showing the average in each $0.1 \mathrm{mag}$ bin. The dark grey symbols indicate the same but after 10 cycles of Sys-Rem, with the black squares the resulting binned average dispersion. The algorithm is more efficient for brighter stars (for which the photometric errors are more dominated by systematic effects). For the brightest stars $(I<15)$ the mean dispersion has decreased from $0.5 \%$

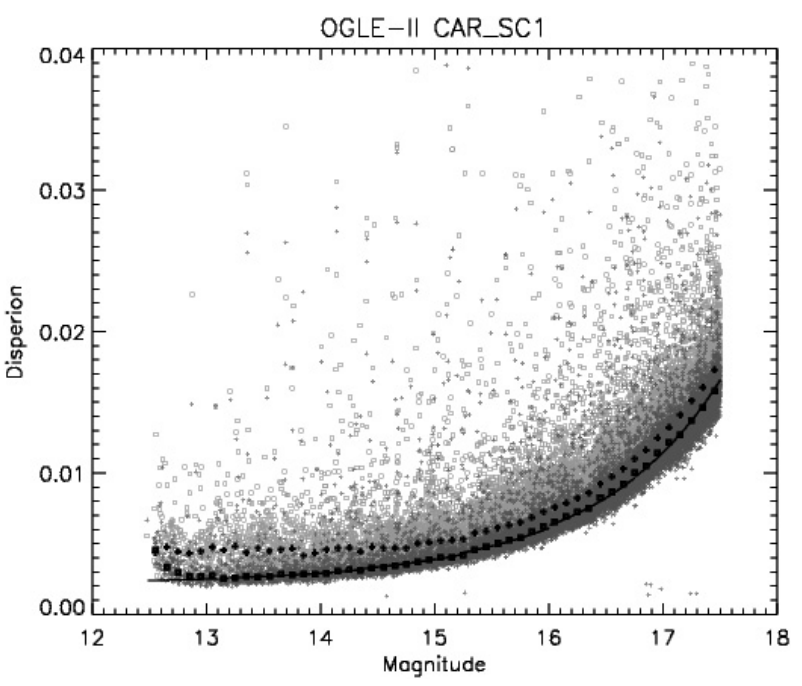

Fig. 1. The dispersion in the stellar light curves as a function of the median magnitude before (light grey points) and after (dark grey points) the use of Sys-Rem. The black points indicate the median dispersion per $0.1 \mathrm{mag}$ bin before and after this detrending correction. It shows that the algorithm is most efficient for the brightest stars. For the bright stars $(I<15)$ the photometric uncertainties are decreased by a factor of $\sim 2$.
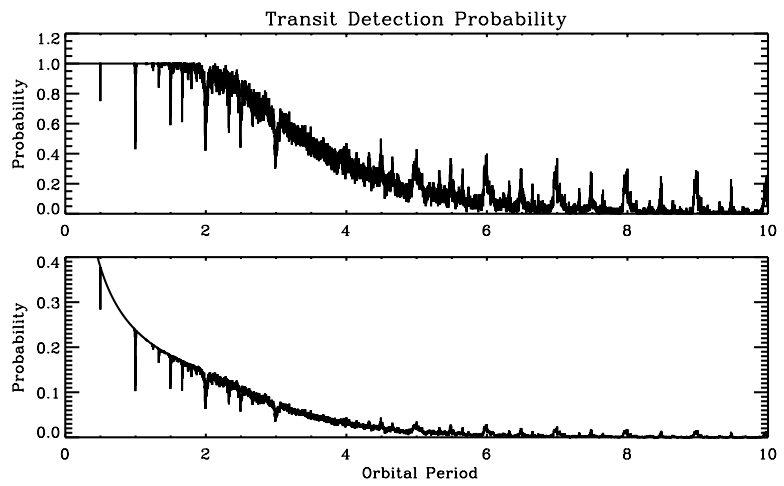

Fig. 2. The transit detection probability for the OGLE-II data as a function of transit period, assuming a Jupiter-size planet transiting a solartype star of $I=16.0$. In the lower panel this probability is multiplied by the probability that a planet actually transits.

before Sys-Rem to $0.2-0.3 \%$ after the use of Sys-Rem, showing the strength of this algorithm. Several of the transits presented in Sect. 3 would not have been found without the use of Sys-Rem. The final mean error as a function of magnitude is fitted with a three parameter function indicated by the solid black line, containing a residual noise term $(0.22 \%)$, and Poisson noise terms from the star and the background sky. The few brightest stars seem to suffer from non-linearity or saturation effects, resulting in an increase of the mean dispersion towards the brightest $I<13$ objects.

After ten cycles of detrending, a box-fitting algorithm was used to search the light curves for periodic transits, based on the Box Least Squares (BLS) method (Kovacs et al. 2002). Transit periods between 0.1 and 10.0 days were searched for using a detection threshold of $8 \sigma$. An estimate of the sensitivity to transiting hot Jupiters as a function of their orbital period is shown in Fig. 2, with in the upper panel the probability to detect a transiting object, and in the lower panel this same probability multiplied by the chance a planet in such an orbit will actually transit its star (assuming a random orbital orientation). For this 
Table 2. Candidate low luminosity and planetary transits in the OGLE2 Carina fields towards the galactic disc.

\begin{tabular}{lllllrrc}
\hline \hline Candidate & OGLE-II Name & $\begin{array}{c}\text { RA(J2000) } \\
\mathrm{h} \mathrm{m} \mathrm{s}\end{array}$ & $\begin{array}{c}\text { Dec }(\mathrm{J} 2000) \\
\mathrm{d} \mathrm{m} \mathrm{s}\end{array}$ & $I$ & $\begin{array}{r}S_{\mathrm{T}} / \sigma \\
\mathrm{mag}\end{array}$ & \multicolumn{1}{c}{$\begin{array}{c}P \\
\text { days }\end{array}$} & $\begin{array}{c}T_{0} \\
(\mathrm{HJD})\end{array}$ \\
\hline OGLE2-TR-L1 & CAR_SC1_59908 & 110551.70 & -613248.7 & 14.780 & 11.8 & $2.7801006 \pm 1.5 \mathrm{e}-5$ & $479.009 \pm 0.0014$ \\
OGLE2-TR-L2 & CAR_SC1_66174 & 110556.28 & -612735.3 & 15.647 & 9.5 & $0.6619433 \pm 1.3 \mathrm{e}-6$ & $477.077 \pm 0.0003$ \\
OGLE2-TR-L3 & CAR_SC1_121984 & 110610.75 & -611452.9 & 15.919 & 16.8 & $1.5841842 \pm 3.0 \mathrm{e}-6$ & $478.074 \pm 0.0008$ \\
OGLE2-TR-L4 & CAR_SC1_138478 & 110650.39 & -615150.3 & 14.831 & 10.3 & $2.0867921 \pm 8.0 \mathrm{e}-6$ & $476.687 \pm 0.0010$ \\
OGLE2-TR-L5 & CAR_SC1_144353 & 110633.74 & -614317.0 & 15.980 & 23.5 & $1.8214055 \pm 4.0 \mathrm{e}-6$ & $476.818 \pm 0.0009$ \\
OGLE2-TR-L6 & CAR_SC1_167415 & 110657.49 & -611719.8 & 15.519 & 9.7 & $0.8177890 \pm 9.0 \mathrm{e}-6$ & $476.864 \pm 0.0004$ \\
OGLE2-TR-L7 & CAR_SC1_169832 & 110651.21 & -611110.3 & 15.340 & 8.1 & $1.8038728 \pm 7.0 \mathrm{e}-6$ & $477.777 \pm 0.0009$ \\
OGLE2-TR-L8 & CAR_SC2_71230 & 110746.96 & -611805.6 & 14.993 & 25.1 & $0.8525568 \pm 1.7 \mathrm{e}-6$ & $477.040 \pm 0.0004$ \\
OGLE2-TR-L9 & CAR_SC2_75679 & 110755.29 & -610846.3 & 13.974 & 15.5 & $2.4855300 \pm 1.7 \mathrm{e}-5$ & $478.661 \pm 0.0012$ \\
OGLE2-TR-L10 & CAR_SC2_78119 & 110753.46 & -610418.2 & 15.890 & 21.1 & $0.8602108 \pm 1.7 \mathrm{e}-6$ & $477.150 \pm 0.0004$ \\
OGLE2-TR-L11 & CAR_SC2_145417 & 110837.25 & -612016.6 & 14.627 & 16.0 & $2.3088322 \pm 7.1 \mathrm{e}-6$ & $478.076 \pm 0.0011$ \\
OGLE2-TR-L12 & CAR_SC2_152898 & 110849.96 & -611051.1 & 15.582 & 12.3 & $3.8237386 \pm 4.2 \mathrm{e}-5$ & $479.461 \pm 0.0019$ \\
OGLE2-TR-L13 & CAR_SC3_155530 & 111035.90 & -603949.1 & 14.848 & 18.9 & $0.8836109 \pm 1.0 \mathrm{e}-6$ & $476.663 \pm 0.0004$ \\
\hline
\end{tabular}

In Col. 1 the candidate name is given, in Col. 2 the OGLE-II name, in Cols. 3 and 4 the RA and Dec (J2000), in Col. 5 the mean $I$ band magnitude, in Col. 6 the signal-to-noise ratio of the transit, and in Cols. 7 and 8 transit period and the time of transit $(-2450000 \mathrm{~d})$ respectively, with their $1 \sigma$ uncertainty.

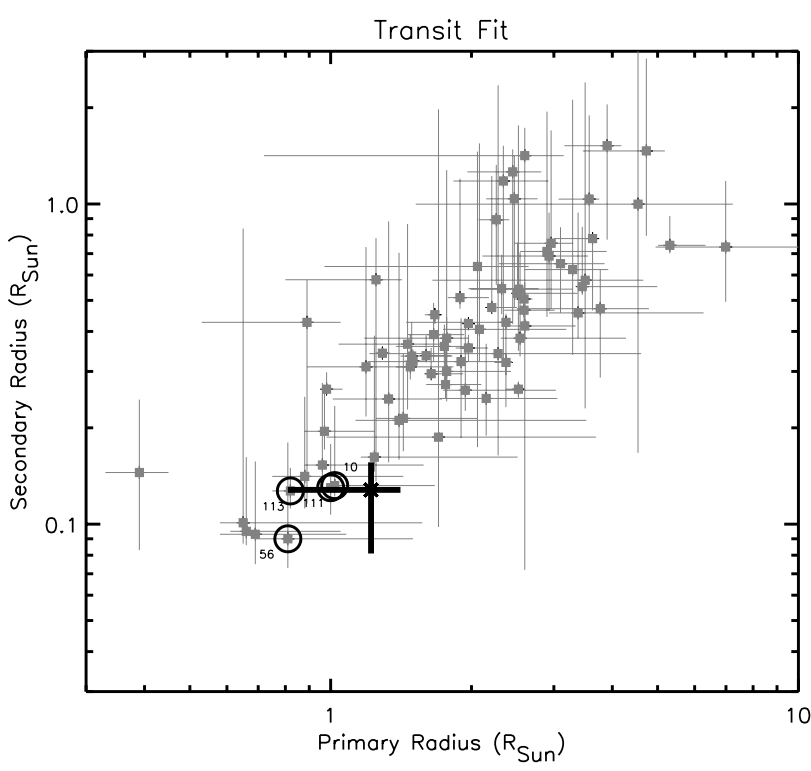

Fig. 3. The radii of the transited and transiting objects from the OGLE-III 2001 and 2002 campaigns, as estimated from least square fitting of the transit shape using the equations of Mandel \& Agol (2002). Only those objects without significant ellipsoidal light variations are shown. The circles indicate the four confirmed planets in these samples. The thick black cross indicates the same for the planetary candidate, OGLE2-TR-L9, presented in this paper.

calculation we assumed only Jupiter-size objects orbiting solar type $\left(I=16.0, \sigma_{I}=0.005\right)$ stars, thus requiring 16 in-transit data points to reach an $8 \sigma$ detection. Note that, in contrast to dedicated transit surveys, these 16 data points will most likely come from 16 individual transits, since the time in between observations is typically much larger ( 1 day) than the transit duration $(\mathrm{a}$ few $\mathrm{h})$. This also means that correlated noise is much less of a problem, with the notable exception for near-integer periods. In the latter case, all data points of a certain period in time will fall within one or a few areas in phase space. Low level stellar variability or slowly varying instrumental instabilities could, if one is not careful, easily lead to spurious transit detections.

Several dozens of eclipsing binaries and low amplitude transiting objects were found among the $\sim 27000$ stars. These transits were least square fitted using the equations of Mandel \& Agol (2002), keeping the orbital inclination fixed at $i=90^{\circ}$. In this way the degeneracy between stellar radius and the transit impact parameter was avoided. By assuming that the primary star is on the main sequence, with $M_{1} / M_{\text {sun }}=R_{1} / R_{\text {sun }}$, this fit subsequently points to lower limits of both the radii of the transited and transiting objects. In addition, a linear limb darkening profile was assumed with a coefficient of $\mu=0.5$. Those systems that were found to have a transiting object with a minimum radius of $R_{2}<2.5 R_{\text {jup }}$ remain transiting planet candidates.

We subsequently searched for ellipsoidal light variations in those remaining planet candidates, since these fluctuations will point to stellar companions: if the secondary object is sufficiently massive and in a sufficiently close-in orbit, tidal effects will deform the primary star. The change in observed solid angle and gravity darkening effects then results in sinusoidal light variations with a period of half the orbital period (Drake 2003; Sirko $\&$ Paczynski 2003). Sinusoidal light variations equal to the transit period are also possible and searched for, either because of possible heating (reflection) effects, or because the actual orbital period is twice as large as assumed, due to the fact that both the primary and secondary eclipses are detected. For all the remaining candidates we also retrieved the $J, H$, and $K$ near-infrared magnitudes from the 2MASS survey (Skrutskie et al. 2006).

\section{Results and discussion}

Our transiting planet candidates are presented in Tables 2 and 3, and in Fig. 4 in the Appendix. Table 2 gives general information and ephemeris of the objects, and Table 3 presents the outcome of the least square fits to the transits and of possible out-of-transit ellipsoidal light variations.

We found a total of 13 interesting transiting objects; 7, 5, and 1 in fields SC1, SC2, and SC3 respectively. All show a transit shape consistent with a secondary object of possibly planetary radius. Only one object, OGLE2-TR-L9 (CAR_SC2_75679) shows no sign of out-of-transit ellipsoidal light variations and is our best candidate (see below). Seven out of thirteen objects show clear $(>4 \sigma)$ sinusoidal variations indicative of massive companions. Three others, OGLE2-TR-L7, OGLE2-TR-L12, and OGLE-TR-L1 show hints of these variations at 2.8 $\sigma, 3.6 \sigma$, 
Table 3. The results of least-squared fitting of the folded light curves.

\begin{tabular}{|c|c|c|c|c|c|c|c|c|c|}
\hline 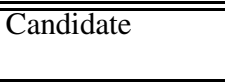 & $\begin{array}{l}\text { Depth } \\
\%\end{array}$ & $\overline{\overline{\mathrm{D} / \mathrm{P}}}$ & $\begin{array}{c}R_{1 \text { min }} \\
R_{\text {Sun }} \\
\end{array}$ & $\begin{array}{c}R_{2 \min } \\
R_{\text {Jup }} \\
\end{array}$ & $\begin{array}{r}E_{1} \\
10^{-3}\end{array}$ & $\begin{array}{c}E_{2} \\
10^{-3} \\
\end{array}$ & $\overline{\bar{J}}$ & $\overline{\bar{H}}$ & $\overline{\bar{K}}$ \\
\hline OGLE2-TR-L1 & $1.2 \pm 0.1$ & $0.038 \pm 0.003$ & 1.0 & 1.1 & $+0.2 \pm 0.3$ & $-1.1 \pm 0.3$ & $14.009 \pm 0.026$ & $13.727 \pm 0.022$ & $13.606 \pm 0.044$ \\
\hline OGLE2-TR-L2 & $0.6 \pm 0.1$ & $0.119 \pm 0.005$ & 1.3 & 1.0 & $-2.2 \pm 0.5$ & $-0.3 \pm 0.4$ & $14.175 \pm 0.041$ & $13.688 \pm 0.060$ & $13.267 \pm 0.044$ \\
\hline OGLE2-TR-L3 & $3.4 \pm 0.1$ & $0.062 \pm 0.003$ & 1.2 & 2.2 & $+0.2 \pm 0.8$ & $-1.5 \pm 0.8$ & $14.367 \pm 0.032$ & $13.954 \pm 0.058$ & $13.582 \pm 0.054$ \\
\hline OGLE2-TR-L4 & $0.9 \pm 0.1$ & $0.056 \pm 0.003$ & 1.3 & 1.3 & $-2.3 \pm 0.3$ & $+0.3 \pm 0.3$ & $14.051 \pm 0.029$ & $13.788 \pm 0.044$ & $13.548 \pm 0.051$ \\
\hline OGLE2-TR-L5 & $3.2 \pm 0.1$ & $0.049 \pm 0.002$ & 0.9 & 1.7 & $-0.0 \pm 0.5$ & $-2.0 \pm 0.5$ & $15.094 \pm 0.057$ & $14.754 \pm 0.068$ & $14.546 \pm 0.119$ \\
\hline OGLE2-TR-L6 & $0.7 \pm 0.1$ & $0.102 \pm 0.003$ & 1.3 & 1.1 & $-2.4 \pm 0.5$ & $-0.9 \pm 0.4$ & $12.849 \pm 0.026$ & $11.537 \pm 0.022$ & $11.035=$ \\
\hline OGLE2-TR-L7 & $0.6 \pm 0.1$ & $0.065 \pm 0.003$ & 1.4 & 1.1 & $-0.7 \pm 0.4$ & $-1.1 \pm 0.4$ & $14.641 \pm 0.065$ & $14.308 \pm 0.081$ & .080 \\
\hline OGLE2-TR-L8 & $1.3 \pm 0.1$ & $0.122 \pm 0.004$ & 1.7 & 2.0 & $-2.8 \pm 0.4$ & $-0.7 \pm 0.3$ & $13.382 \pm 0.026$ & $12.538 \pm 0.027$ & 12.252 \\
\hline OGLE2-TR-L9 & $1.1 \pm 0.1$ & $0.038 \pm 0.004$ & 0.9 & 0.9 & $-0.2 \pm 0.3$ & $+0.3 \pm 0.2$ & $13.508 \pm 0.032$ & $13.246 \pm 0.035$ & $13.117 \pm 0.037$ \\
\hline OGLE2-TR-L10 & $2.7 \pm 0.1$ & $0.077 \pm 0.003$ & 0.9 & 1.5 & $-2.0 \pm 0.5$ & $+0.0 \pm 0.5$ & $15.078 \pm 0.054$ & $14.746 \pm 0.081$ & $14.602 \pm 0.119$ \\
\hline OGLE2-TR-L11 & $1.1 \pm 0.1$ & $0.060 \pm 0.002$ & 1.6 & 1.7 & $-0.9 \pm 0.3$ & $-1.5 \pm 0.2$ & $14.160 \pm 0.063$ & $14.066 \pm 0.082$ & $13.809 \pm 0.074$ \\
\hline OGLE2-TR-L12 & $1.6 \pm 0.1$ & $0.045 \pm 0.005$ & 1.8 & 2.2 & $-1.8 \pm 0.5$ & $-0.4 \pm 0.4$ & $14.707 \pm 0.071$ & $14.391 \pm 0.089$ & $13.829^{\dagger}$ \\
\hline OGLE2-TR-L13 & $1.1 \pm 0.1$ & $0.073 \pm 0.003$ & 0.8 & 0.9 & $-2.0 \pm 0.3$ & $+0.5 \pm 0.3$ & $14.127 \pm 0.079$ & $13.726 \pm 0.136$ & $13.670 \pm 0.137$ \\
\hline
\end{tabular}

In Col. 1 the candidate name is given, in Col. 2 the depth of the transit, in Col. 3 the length of the transit, in Cols. 4 and 5 the fitted minimum radius of the primary and secondary object, assuming a zero impact parameter for the transit and assuming that the primary is on the main sequence. Columns 6 and 7 give the fitted amplitude of possible ellipsoidal light variation, with periods half and equal to the orbital period respectively. Columns 8-10 show the 2MASS $J, H$, and $K$ magnitudes.

and $3.7 \sigma$ respectively, and their transiting objects are therefore also unlikely to be planets.

One object, OGLE-TR-L3, is also of particular interest (see Fig. 4). It shows a significant secondary eclipse, only identifiable because it is not at half time between transits, but at phase -0.37 . This must be due to a non-circular orbit of the transiting object. The timing of the secondary eclipse implies that the orbital eccentricity is $e>0.2$. This is quite remarkable since the orbital period is only $P=1.58$ days, implying a short tidal circulization time scale. This means that it should be a recently formed binary, or alternatively, that a third body is present in this system.

\section{OGLE2-TR-L9: An excellent exoplanet candidate}

The transit and ellipsoidal light curve analysis shows that OGLE2-TR-L9, with an orbital period of $P=2.4855$ days, is our best exoplanet candidate. The least square fit to its transit yields a lower limit to the radius of the primary of $0.9 R_{\text {sun }}$, and to the radius of the secondary of $0.9 R_{\text {jup }}$. The optical to nearinfrared colours of the star, $I-J=0.466$ and $J-K=0.391$, are that of a mid-G star, and therefore consistent with the $0.9 R_{\text {sun }}$ radius of the primary. We also performed a least square fit using the equations of Mandel \& Agol (2002), leaving the impact parameter, $\kappa$, as a free variable. If the transit was at a high impact parameter (making the radii of the primary and secondary more uncertain), it would form a more "V" shaped transit, with the ingress and egress times being relatively large with respect to the flatter mid-transit part. In contrast, the transit is best fitted with a low impact parameter of $\kappa<0.7\left(\kappa=0.6_{-0.6}^{+0.1}, R_{1}=1.22_{-0.41}^{+0.19}\right.$ and $\left.R_{2}=0.122_{-0.041}^{+0.032}\right)$, resulting in a planet radius of $<1.5 R_{\text {jup }}$. Hence, this analysis makes it very likely we are indeed dealing with a planet size object.

To assess the probability that OGLE2-TR-L9 is of planetary nature, we performed the same light curve analysis on all the 117 OGLE-III low amplitude transit candidates from the 2001 and 2002 campaigns as published in Udalski et al. (2002a,c). No ellipsoidal light variations could be found at $>0.2 \%$ amplitude level for 74 out of 117 targets. Of these, only 9 are best fitted with an transiting object with radius $<1.5 R_{\text {jup }}$, of which four turned out to be planets. Therefore, with the additional information from the 2MASS photometry we estimate the likelihood that
OGLE2-TR-L9 is indeed a transiting extrasolar planet at $\sim 50 \%$, and deserves follow-up observations.

The accuracy in the orbital period is such that, now, seven years after the last OGLE-II observations (some 1000 periods later), the uncertainty in the transit timing has increased to about $30 \mathrm{~min}$. First the transit should be searched for and the transit shape measured at 30-40 $\sigma$ precision with a 1-2 m telescope. If the transit shape at high signal-to-noise, and spectral stellar typing is still consistent with a transit of planetary nature, radial velocity observations of this $\operatorname{star}(I=13.97)$ should be performed to measure the mass of the secondary. Plans for these follow-up observations are in place.

\section{Conclusions}

In this paper we searched the online database of the second phase of the OGLE project (OGLE-II; 1997-2000) for transiting extrasolar planets, concentrating on the galactic disc fields in the Carina constellation. The main goals were, next to finding possibly more transiting exoplanets, to investigate whether such a data set with a very different cadence compared to typical transit surveys, is actually sensitive to low amplitude transits.

We found that detrending the data using the Sys-Rem algorithm significantly reduced the scatter in the light curves by about a factor two, down to $0.2 \%$. About 27000 stars were searched for transits with periods between 0.5 and 5 days using the BLS algorithm, and several dozens of eclipsing binaries were found. Thirteen stars were found to exhibit low amplitude transits, of which most show significant ellipsoidal light variability indicative of tidal interactions and stellar mass secondaries. One star, OGLE2-TR-L9 ( $P=2.4855$ days), shows a transit shape consistent with a Jupiter size object orbiting a solar type star, also consistent with its optical to near-infrared colours. Comparing this candidate to those from the dedicated OGLE-III transit survey campaigns, we estimate $a \sim 50 \%$ probability for this to be a genuine transiting planet, deserving follow-up observations.

Acknowledgements. We thank the OGLE-team, in particular M. Szymanski, for making the OGLE-II database available online. This publication also makes use of data products from the Two Micron All Sky Survey, which is a joint project of the University of Massachusetts and the Infrared Processing and Analysis Center/California Institute of Technology, funded by the National Aeronautics and Space Administration and the National Science Foundation. The Digitized 

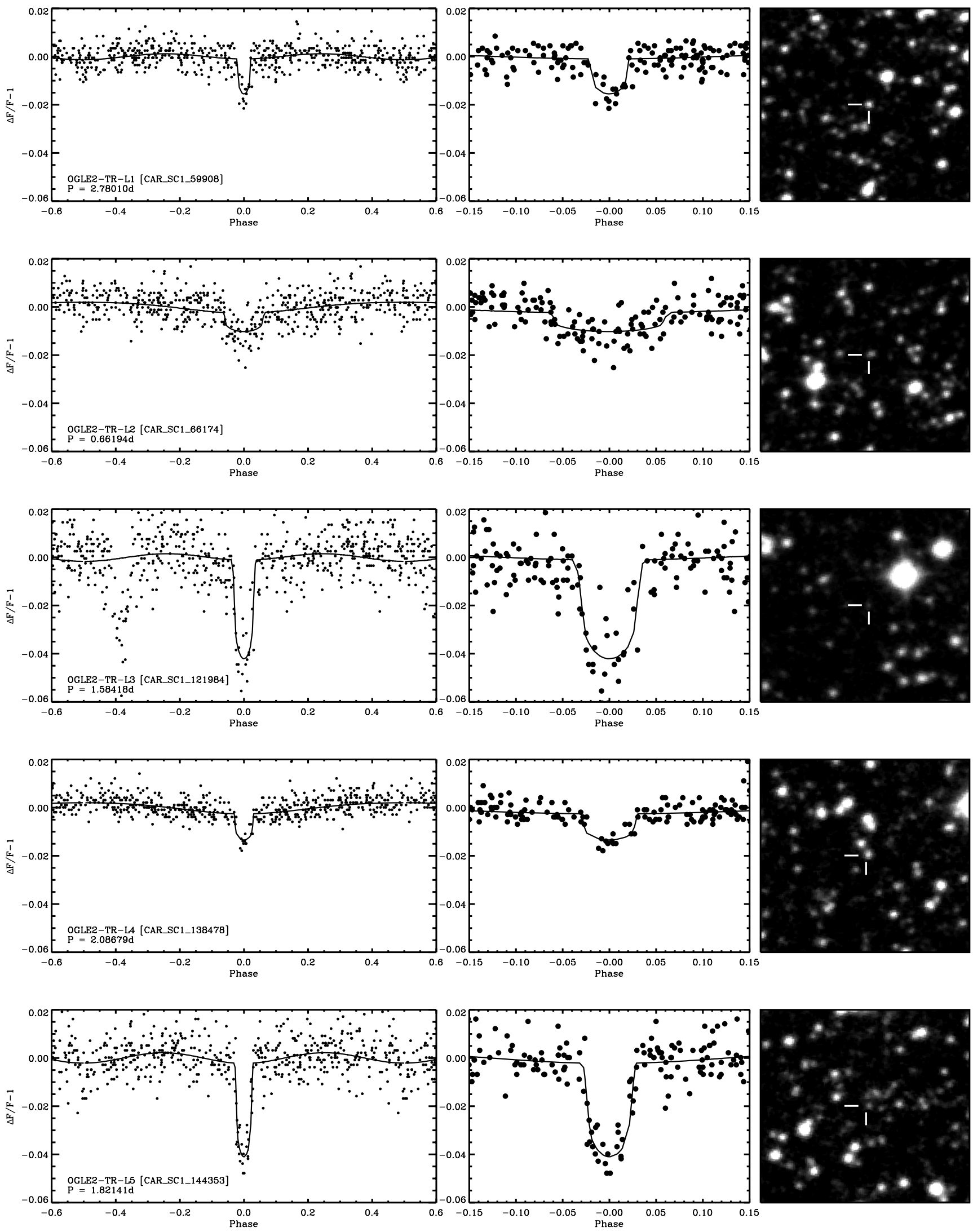

Fig. 4. The folded light curves and finding charts of our transit candidates. 

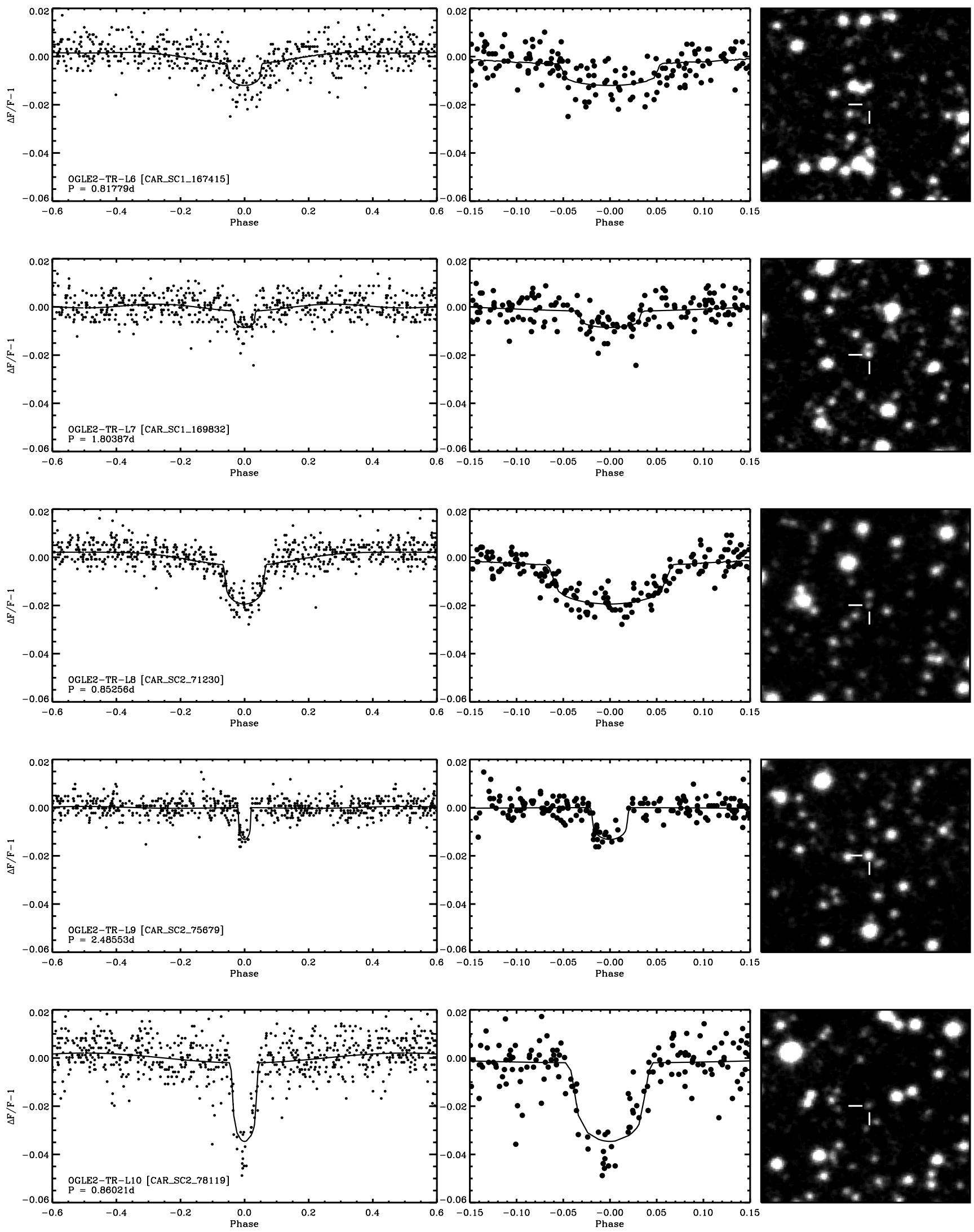

Fig. 4. continued. 

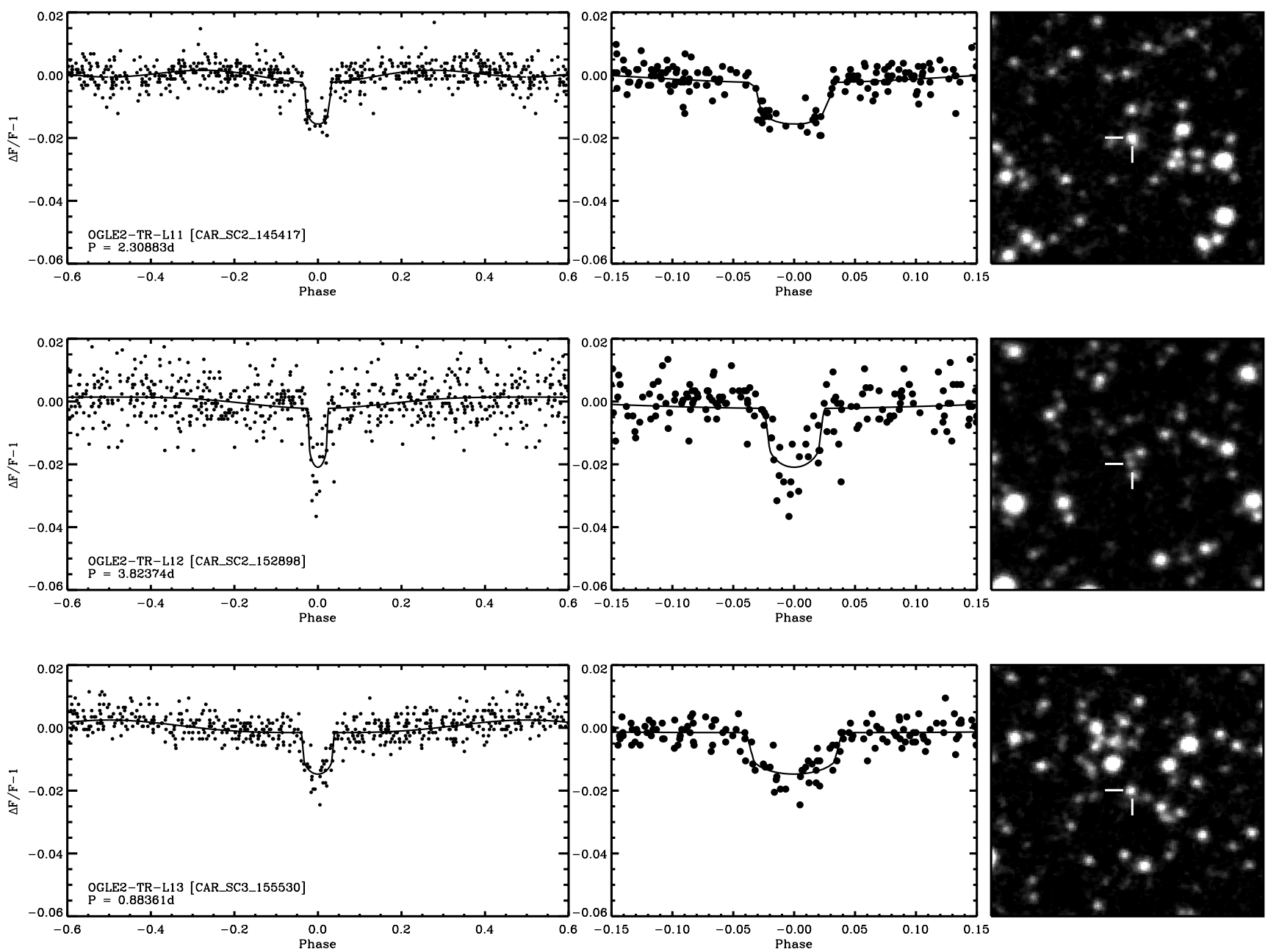

Fig. 4. continued.

Sky Survey was produced at the Space Telescope Science Institute under US Government grant NAG W-2166. The images of these surveys are based on photographic data obtained using the Oschin Schmidt Telescope on Palomar Mountain and the UK Schmidt Telescope. The plates were processed into the present compressed digital form with the permission of these institutions.

\section{Appendix A}

In this appendix we present the folded light-curves of the thirteen low amplitude transiting candidates. The left panel shows for each star the complete light-curve, while the middle panel zooms in on $-0.15<$ phase $<0.15$. The right panel is for each star an extract from the Digitized Sky Survey, measuring $2 \times 2$ arcmin.

\section{References}

Aigrain, S., \& Pont, F. 2007, MNRAS, 378, 741

Alard, C. 2000, A\&AS, 144, 363

Alard, C., \& Lupton, R. H. 1998, ApJ, 503, 325

Afonso, C., \& Henning, Th. 2007, in the proceedings of Transiting Extrasolar Planet Workshop, ed. C. Afonso, D. Weldrake, \& Th. Henning, ASP Conf. Ser. 366,326

Bakos, G. Á., Noyes, R. W., Kovács, G., et al. 2007, ApJ, 656, 552

Bayliss, D. D. R., \& Sackett, P. D. 2007, in the proceedings of Transiting Extrasolar Planet Workshop, ed. C. Afonso, D. Weldrake, \& Th. Henning, ASP Conf. Ser. 366, 320

Bouchy, F., Pont, F., Melo, C., et al. 2005, A\&A, 431, 1105
Charbonneau, D., Brown, T. M., Noyes, R. W., \& Gilligand, R. L. 2002, ApJ 568,377

Charbonneau, D., Allen, L., Megeath, S., et al. 2005, ApJ, 626, 523

Collier Cameron, A., Pollacco, D., Street, R. A., et al. 2006, MNRAS, 373, 799

Collier Cameron, A. C., Bouchy, F., Hébrard, G., et al. 2007, MNRAS, 375, 951

Deming, D., Seager, S., Richardson, J., \& Harrington, J. 2005, Nature , 434, 740

Drake, A. J. 2003, ApJ, 589, 1020

Gaudi, B. S. 2006 [arXiv: astro-ph/0612141]

Kovács, G., Zucker, S., \& Mazeh, T. 2002, A\&A, 391, 369

Mandel, K., \& Agol, E. 2002, ApJ, 580, L171

McCullough, P. R., Stys, J. E., Valenti, J. A., et al. 2005, PASP, 117, 783

O'Donovan, F. T., Charbonneau, D., Bakos, G. Á., et al. 2007, ApJ, 663, L37

Pont, F., Bouchy, F., Melo, C., et al. 2005, A\&A, 438, 1123

Pont, F., Zucker, S., \& Queloz, D. 2006, MNRAS, 373, 231

Sato, B., Fischer, D. A., Henry, G. W., et al. 2005, ApJ, 633, 465

Sirko, E., \& Paczyński, B. 2003, ApJ, 592, 1217

Skrutskie, M. F., Cutri, R. M., Stiening, R., et al. 2006, AJ, 131, 1163

Snellen, I. A. G. 2004, MNRAS, 353, L1

Steffen, J. H., Gaudi, B. S., Ford, E. B., Agol, E., \& Holman, M. J. 2007, [arXiv:0704.0632]

Szymanski, 2005, Acta Astron., 55

Tamuz, O., Mazeh, T., \& Zucker, S. 2005, MNRAS, 356, 1466

Udalski, A., Kubiak, M., \& Szymanski, M. 1997, Acta Astron., 47, 319

Udalski, A., Szymanski, M., Kubiak, M., et al. 1998, Acta Astron., 48, 147

Udalski, A., Paczynski, B., Zebrun, K., et al. 2002a, Acta Astron., 52, 1

Udalski, A., Szewczyk, O., Zebrun, K., et al. 2002b, Acta Astron., 52, 317

Udalski, A., Zebrun, K., Szymanski, M., et al. 2002c, Acta Astron., 52, 115

Udalski, A., Szymanski, M. K., Kubiak, M., et al. 2004, Acta Astron., 54, 313

Winn, J. N. 2007, in the proceedings of Transiting Extrasolar Planet Workshop, ed. C. Afonso, D. Weldrake, \& Th Henning, ASP Conf. Ser. 366, 170 